\title{
Are alternative antibiotics needed for antibiotic-nonresponsive Mycoplasma pneumoniae pneumonia?
}

\author{
Eun-Ae Yang, $M D^{1,2}$, Kyung-Yil Lee, MD ${ }^{1,2}$ \\ ${ }^{1}$ Department of Pediatrics, College of Medicine, The Catholic University of Korea, Seoul, Korea; ${ }^{2}$ Department of Pediatrics, The Catholic University of Korea Daejeon \\ St. Mary's Hospital, Daejeon, Korea
}

Mycoplasma pneumoniae (MP) is a major etiologic agent of community-acquired pneumonia in children and young adults, and MP pneumonia has occurred as cyclic nationwide epidemics of 3-to 4-year intervals in Korea. The recent 2015-2016 epidemic in Korea reported that macrolide-resistant MP (MRMP) strains were detected in over $80 \%$ of cases. Thus, treatment strategies for patients with MRMP strains have emerged in Korea and other countries. For this issue, Yang reviewed the treatment guidelines for severe patients infected with MRMP strains. ${ }^{1)}$ Patients who did not respond to macrolide antibiotics (persistent fever for 48-72 hours or disease progression) were treated with secondline antibiotics such as tetracyclines or fluoroquinolones. The author indicated that these antibiotics were not approved to use for children because of the potential risk of complications and the guidelines were based on expert opinions without evidence-based results. $^{1)}$

Pneumonia caused by MP, previously known as atypical pneumonia or pleuropneumonia-like organism pneumonia, was first noticed by researchers in the 1940s. As MP is an extremely small microorganism having a fastidious growing nature, its identification as a pathogenic agent was achieved in the 1960s. ${ }^{2}$ In vitro, $\mathrm{MP}$ is very sensitive to antibiotics, including macrolides, tetracyclines, and fluoroquinolones. A few investigators in the early 1960s reported that erythromycin and tetracycline were effective against MP in young adults. Accordingly, physicians have prescribed antibiotics to patients with MP pneumonia. Conversely, earlier animal studies revealed that early antibiotic administration after MP inoculation could not prevent the disease and that antibiotic therapy could not remove MP from the MP-infected host. ${ }^{3)}$ Few intact MP pathogens were identified in pathologic lesions in experimental animal and human MP infections. ${ }^{4)}$ These findings suggest that the pathogenesis of MP pneumonia may not be directly associated with pathogen-induced cytopathy.

MP pneumonia is a self-limited disease, though some patients show a protractive clinical course. Antibiotic-nonresponsive or refractory MP pneumonia is believed to be caused by antibioticresistant MP strains, mainly MRMP. However, a small propor- tion of MP pneumonia patients show progressive pneumonia or extrapulmonary manifestations despite the use of susceptible antibiotics during MP pneumonia epidemics. ${ }^{5)}$ Empiric antibiotic coverage for MP has not shown clinical efficacy on outcomes in children with lower respiratory tract infections caused by MP.6) Although children with MRMP infections treated with tetracyclines or quinolones reportedly show more rapid defervescence, outcomes such as the absence of fatality or severe morbidity rates did not differ between treatment groups. Many patients who received alternative antibiotics might be concurrently treated with corticosteroids.7) Clinical parameters reportedly did not differ between MRMP and macrolide-susceptible MP pneumonia patients, while some MRMP pneumonia patients responded to macrolides, suggesting that macrolide resistance did not contribute to the clinical severity of MRMP pneumonia. ${ }^{8)}$ However, few controlled studies have presented clear evidence of the effectiveness of antibiotics on MP infections in children. This may be due in part to obstacles such as a lack of early diagnostic tools for patient collection, difficulty creating an acceptable control group without antibiotics, or a problem with outcome measures for this mild and self-limiting disease.

Antibiotics have a limited effect on the natural course of acute viral infections, infection-related immune-mediated diseases such as acute poststreptococcal glomerulonephritis and Kawasaki disease, and extrapulmonary manifestations in MP infection such as hemolytic anemia and neurologic complications. It is acceptable to use early empiric antibiotics for patients with any pneumonia, especially severe pneumonia in elderly persons with multiple comorbidities or children with immunedeficient states. However, outcomes of pneumonia patients may depend on the host's immune status and not on antibiotic treatment because the substances inducing lung injury are not pathogens themselves; instead, they are other smaller substances including pathogen-associated molecular patterns (PAMPs) and/ or damage-associated molecular patterns (DAMPs).

There have been few well-controlled studies on the efficacy of immune-modulators such as corticosteroids or intravenous immunoglobulin on MP pneumonia, but corticosteroids effec-

Corresponding author: Kyung-Yil Lee, MD. Department of Pediatrics, The Catholic University of Korea Daejeon St. Mary's Hospital, 64 Daeheung-ro, Jung-gu, Daejeon 34943, Korea 
tively initiate the rapid clinical and chest radiography improvements in children and adults with severe MSMP and MRMP pneumonia. ${ }^{4,5)}$ The epidemiological characteristics of MP infection, such as cyclic epidemics and case predominance in young children, are similar to certain respiratory viral infections such as measles in the pre-vaccine era, ${ }^{4,5)}$ and MP can invade into the host cells and spread to other organ cells like viruses.9) Therefore, it is possible that inflammation-inducing substances in MP infection are produced when pathogens replicate within host cells, including toxins such as community-acquired respiratory distress syndrome toxins and PAMPs; substances from infected host cell origin, including DAMPs; and proinflammatory cytokines and proteolytic enzymes from activated immune cells. The host's immune system is proposed to control these diverse substances by their size and biochemical characteristics. ${ }^{10)}$ Since the progression of pneumonia is associated with an excessive immune reaction against the MP infection, alternative antibiotics may have a limited effect on rapidly progressive and severe MRMP pneumonia. Thus, well-controlled studies are needed of corticosteroid and antibiotic use in the treatment of MP pneumonia.

See the article "Benefits and risks of therapeutic alternatives for macrolide resistant Mycoplasma pneumoniae pneumonia in children" in Volume 62 on page 199.

\section{Conflicts of interest}

No potential conflict of interest relevant to this article was reported.

\section{References}

1. Yang HJ. Benefits and risks of therapeutic alternatives for macrolide resistant Mycoplasma pneumoniae pneumonia in children. Korean J Pediatr 2019;62:199-205.

2. Saraya T. The history of Mycoplasma pneumoniae pneumonia. Front Microbiol 2016;7:364.

3. McCracken GH Jr. Current status of antibiotic treatment for Mycoplasma pneumoniae infections. Pediatr Infect Dis 1986;5:167-71.

4. Lee KY. Pediatric respiratory infections by Mycoplasma pneumoniae. Expert Rev Anti Infect Ther 2008;6:509-21.

5. Yang EA, Kang HM, Rhim JW, Kang JH, Lee KY. Early corticosteroid therapy for Mycoplasma pneumoniae pneumonia irrespective of used antibiotics in children. J Clin Med 2019;8:726.

6. Gardiner SJ, Gavranich JB, Chang AB. Antibiotics for community-ac quired lower respiratory tract infections secondary to Mycoplasma pneumoniae in children. Cochrane Database Syst Rev 2015;1:CD004875.

7. Okada T, Morozumi M, Tajima T, Hasegawa M, Sakata H, Ohnari S, et al. Rapid effectiveness of minocycline or doxycycline against macrolideresistant Mycoplasma pneumoniae infection in a 2011 outbreak among Japanese children. Clin Infect Dis 2012;55:1642-9.

8. Lee H, Yun KW, Lee HJ, Choi EH. Antimicrobial therapy of macrolideresistant Mycoplasma pneumoniae pneumonia in children. Expert Rev Anti Infect Ther 2018;16:23-34.

9. Hegde S, Hegde S, Spergser J, Brunthaler R, Rosengarten R, ChopraDewasthaly R. In vitro and in vivo cell invasion and systemic spreading of Mycoplasma agalactiae in the sheep infection model. Int J Med Microbiol 2014;304:1024-31.

10. Lee KY. Pneumonia, acute respiratory distress syndrome, and early immune-modulator therapy. Int J Mol Sci 2017;18:388. 